\begin{tabular}{|c|c|}
\hline Title & Spin transport in ferromagnet/semiconductor ferromagnet structures with cubic Dressel haus spin-orbit-interaction \\
\hline Author(s) & Kondo, Kenji \\
\hline Citation & $\begin{array}{l}\text { Journal of A pplied Physics, 111(7), 07C 713 } \\
\text { https://doi.org/10.1063/1.3677799 }\end{array}$ \\
\hline Issue Date & 2012-04-01 \\
\hline Doc URL & http:/hdl.handle.net/2115/49379 \\
\hline Rights & $\begin{array}{l}\text { Copyright } 2012 \text { A merican Institute of Phy sics. This article may be downloaded for personal use only. A ny other use } \\
\text { requires prior permission of the author and the A merican Institute of Physics. The following article appeared in J. A ppl. } \\
\text { Phys. } 111,07 C 713 \text { (2012) and may be found at https://dx.doi.org/10.1063/1.3677799 }\end{array}$ \\
\hline Type & article \\
\hline File Information & JAP111-7_07C713.pdf \\
\hline
\end{tabular}

Instructions for use 


\section{AIP Appilied Physics}

\section{Spin transport in ferromagnet/semiconductor/ferromagnet structures with cubic Dresselhaus spin-orbit-interaction}

Kenji Kondo

Citation: J. Appl. Phys. 111, 07 C713 (2012); doi: 10.1063/1.3677799

View online: http://dx.doi.org/10.1063/1.3677799

View Table of Contents: http://jap.aip.org/resource/1/JAPIAU/v111/i7

Published by the American Institute of Physics.

\section{Related Articles}

Origin of magneto-optic enhancement in CoPt alloys and Co/Pt multilayers Appl. Phys. Lett. 100, 232409 (2012)

Reproducible domain wall pinning by linear non-topographic features in a ferromagnetic nanowire Appl. Phys. Lett. 100, 232402 (2012)

L10-FePt based exchange coupled composite films with soft [Co/Ni]N multilayers

J. Appl. Phys. 111, 103916 (2012)

Transport and magnetic properties of the Co2MnSi/Al/Co2MnSi trilayer

Appl. Phys. Lett. 100, 222407 (2012)

Spin-orbit field switching of magnetization in ferromagnetic films with perpendicular anisotropy Appl. Phys. Lett. 100, 212405 (2012)

\section{Additional information on J. Appl. Phys.}

Journal Homepage: http://jap.aip.org/

Journal Information: http://jap.aip.org/about/about_the_journal

Top downloads: http://jap.aip.org/features/most_downloaded

Information for Authors: http://jap.aip.org/authors

\section{ADVERTISEMENT}

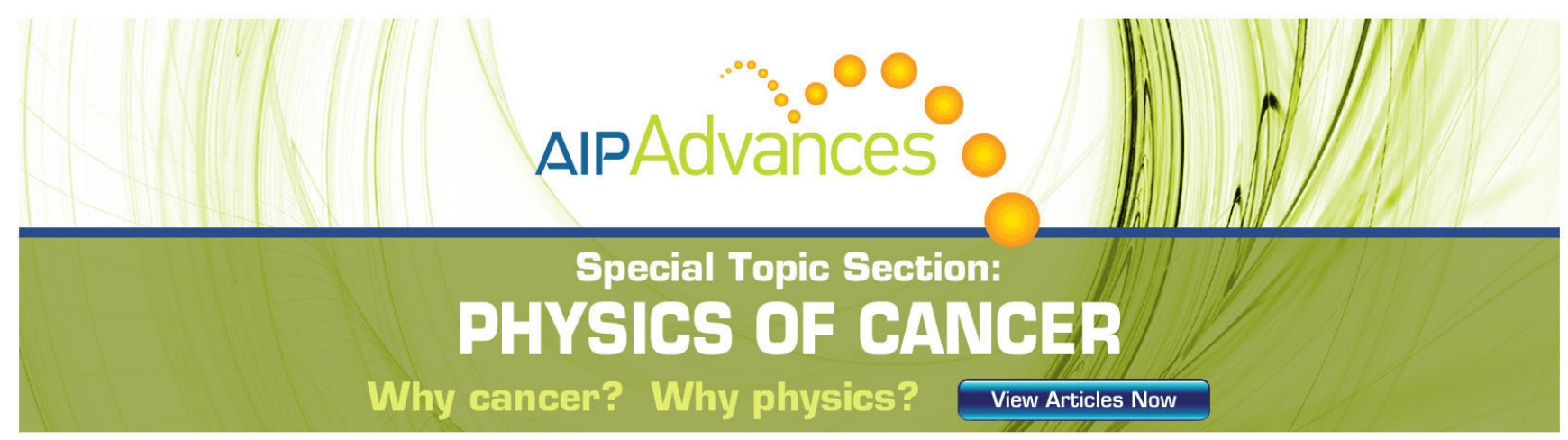




\title{
Spin transport in ferromagnet/semiconductor/ferromagnet structures with cubic Dresselhaus spin-orbit-interaction
}

\author{
Kenji Kondo ${ }^{\text {a) }}$ \\ Laboratory of Quantum Electronics, Research Institute for Electronic Science, Hokkaido University, Sapporo, \\ Hokkaido 001-0020, Japan
}

(Presented 2 November 2011; received 14 September 2011; accepted 18 November 2011; published online 8 March 2012)

\begin{abstract}
We have investigated the spin transport in ferromagnet (FM)/semiconductor (SC)/ferromagnet (FM) structures with a central SC barrier region exhibiting cubic Dresselhaus spin-orbit-interaction (SOI). The energy profile of the barrier is assumed to be a square with height $V$ and thickness $d$ along $z$-direction. The magnetoresistance (MR) ratio has been calculated for three different barriers, GaAs, GaSb, and GaAs without SOI as a function of barrier thickness. We have found that the MR ratio has a negative value for GaAs barrier with SOI except for very thin barrier thickness. In the case of $\mathrm{GaSb}$ barrier, the MR ratio changes sign from negative to positive with increasing the barrier thickness. Also, we have calculated the MR ratio with changing the spin coupling constant. (C) 2012 American Institute of Physics. [doi:10.1063/1.3677799]
\end{abstract}

\section{INTRODUCTION}

One of the main purposes in the field of spintronics is to make spin transistors with a ferromagnet (FM)/semiconductor $(\mathrm{SC}) /$ ferromagnet $(\mathrm{FM})$ structure. It is very important to investigate the spin transport in FM/SC/FM structures theoretically to realize the spin transistors. ${ }^{1,2}$ The first proposal of the spin transistor was done by Datta and Das. ${ }^{3}$ In this transistor, the spin direction of injected electrons is controlled by adjusting the Rashba spin-orbit-interaction (SOI) by gate voltage. Another proposed device is the spin MOSFET. $^{4}$ In recent years, there have been many successful experiments demonstrating a spin-polarized current through the contact of $\mathrm{Fe} / \mathrm{GaAs}$. In this situation, some experiments of tunneling in FM/SC/FM structures have been reported. ${ }^{5,6}$ One of these experiments reports that a negative value of magnetoresistance (MR) is observed that indicates a negative spin-filtering effect. Recently, we have also proposed a spin quantum cross structure (SQCS) device toward novel spin transistors. $^{7-9}$ In this paper, we have generally investigated the spin transport in FM/SC/FM structures with a central SC barrier region exhibiting cubic Dresselhaus spin-orbit-interaction (SOI). The following results can be useful for designing spin transistors and interpreting many experimental results. Also they can be applied for realizing spin transistors using the SQCS devices.

\section{THEORY AND RESULTS}

We study the transmission of spin $\sigma(\uparrow, \downarrow)$ electrons with the initial wave vector $\boldsymbol{k}_{\sigma}=\left(\boldsymbol{k}_{\| \boldsymbol{\sigma}}, k_{z \sigma}\right)$ in the ferromagnet through the SC barrier region exhibiting cubic Dresselhaus SOI. The model used in the calculation is shown in Fig. 1. The energy profile of the barrier is assumed to be a square with height $V$ and thickness $d$ along $z$-direction. Then, the barrier is described by the function $V(z)=V(\Theta(z)$

a)Electronic mail: kkondo@es.hokudai.ac.jp.
$-\Theta(z-d))$, where $\Theta(z)$ is the step function. The coordinate axes are chosen as shown in Fig. 1. They are assumed to be parallel to the cubic crystallographic axes [100], [010], [001], respectively. The model Hamiltonian in the region $i$ $(i=1,2,3)$ reads $^{10}$

$$
\begin{gathered}
H^{(i)}=H_{0}^{(i)}+H_{\mathrm{D}}^{(i)}+V(z), \\
H_{0}^{(i)}=\frac{\hbar^{2} \boldsymbol{k}_{\sigma}^{2}}{2 m_{i}^{*}}-\frac{\Delta_{i}}{2} \boldsymbol{n}_{i} \cdot \tau, \\
H_{\mathrm{D}}^{(i)}=\gamma_{i}\left(\tau_{x} k_{x \sigma}-\tau_{y} k_{y \sigma}\right) k_{z \sigma}^{2},
\end{gathered}
$$

where $\hbar$ is the Planck constant divided by $2 \pi . m_{i}^{*}$ is the effective mass in the left ferromagnet $(i=1)$, semiconductor $(i=2)$, and right ferromagnet $(i=3)$ regions, respectively. $\Delta_{i}$ is the exchange energy in the region $i$, and the exchange energy $\Delta_{2}$ in the region 2 is zero. The unit vector $\boldsymbol{n}_{i}$ defines the magnetization direction, $\boldsymbol{n}_{1}=(0,1,0)$, $\boldsymbol{n}_{2}=(0,0,0), \boldsymbol{n}_{3}=(0, \cos \theta,-\sin \theta)$. We take the metal Fe as both the left and the right ferromagnets. The Fermi momenta of the majority spin and minority spin of $\mathrm{Fe}$ are chosen as $k_{F \uparrow}=1.05 \times 10^{10} \mathrm{~m}^{-1}$ and $k_{F \downarrow}=0.44 \times 10^{10} \mathrm{~m}^{-1}$,

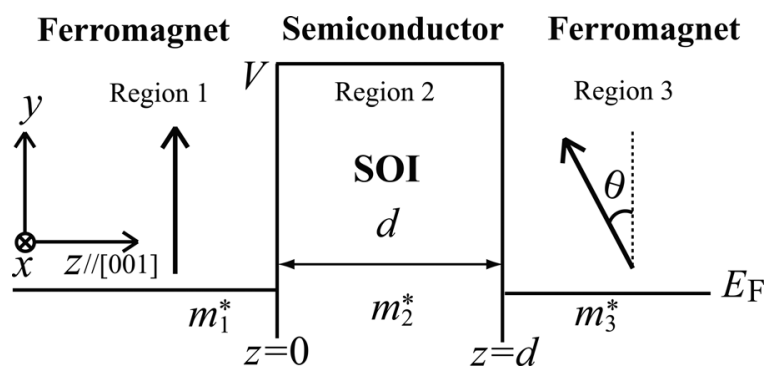

FIG. 1. A schematic illustration of a model of FM/SC/FM structures. Electrons with the wave vector $\boldsymbol{k}_{\sigma}=\left(k_{x \sigma}, k_{y \sigma}, k_{z \sigma}\right)=\left(\boldsymbol{k}_{\| \boldsymbol{\sigma}}, k_{z \sigma}\right)$ transmit through the potential barrier with height $V$ of width $d$ along $z$. The arrows show the magnetization direction in region 1 and region 3. 
respectively. ${ }^{11}$ The band of itinerant d-like electrons of Fe is assumed to be parabolic with the free electron mass $\left(m_{1}^{*}=m_{3}^{*}=m_{e}\right){ }^{12} \gamma_{i}$ is the spin-orbit coupling constant in the region $i$. In this model, only $\gamma_{2}$ is a finite value of $\gamma$, and the others are zero. $\tau_{x}$ and $\tau_{y}$ are $x$-component and $y$-component of the vector of Pauli matrices $\tau$, respectively. The model Hamiltonian in region 2 is explicitly given by

$$
H^{(2)}=\frac{\hbar^{2} \boldsymbol{k}_{\sigma}^{2}}{2 m_{2}^{*}}+\gamma\left(\tau_{x} k_{x \sigma}-\tau_{y} k_{y \sigma}\right) k_{z \sigma}^{2}+V .
$$

The Hamiltonian of Eq. (4) can be diagonalized using the following spinors:

$$
\chi_{\sigma \pm}^{(2)}=\frac{1}{\sqrt{2}}\left(\begin{array}{c}
1 \\
\mp e^{-i \varphi_{\sigma}}
\end{array}\right),
$$

where $\varphi_{\sigma}=\tan ^{-1}\left(k_{y \sigma} / k_{x \sigma}\right)$. These represent the spinstates "+" and "-" in the SC barrier with SOI for incoming electrons with spin $\sigma$. Both spinstates are shown in Figs. 2(a) and 2(b), respectively. The corresponding eigen energies satisfy$\operatorname{ing} H^{(2)} x_{\sigma \pm}^{(2)}=E_{\sigma \pm}^{(2)} x_{\sigma \pm}^{(2)}$ are given by

$$
E_{\sigma \pm}^{(2)}=\frac{\hbar^{2} \boldsymbol{k}_{\sigma}^{2}}{2 m_{2}^{*}} \pm \gamma k_{\| \sigma} k_{z \sigma}^{2}+V,
$$

where $k_{\| \sigma}=\sqrt{k_{x \sigma}^{2}+k_{y \sigma}^{2}}$ is the in-plane wave vector magnitude. The general solutions of the Schrödinger equation $H^{(2)} \Phi_{\sigma \pm}^{(2)}=E_{\sigma \pm}^{(2)} \Phi_{\sigma \pm}^{(2)}$ are of the following form:

$$
\Phi_{\sigma \pm}^{(2)}(\boldsymbol{r})=\exp \left(i \boldsymbol{k}_{\| \sigma} \cdot \boldsymbol{r}_{\|}\right) \phi_{\sigma \pm}^{(2)}(z) \chi_{\sigma \pm}^{(2)},
$$

where $\boldsymbol{r}=(x, y, z), \boldsymbol{k}_{\|}=\left(k_{x}, k_{y}\right)$, and $\boldsymbol{r}_{\|}=(x, y)$. The functions $\phi_{\sigma}^{(1,3)}(z)$ 's in the ferromagnet regions 1 and 3 also have the same forms with different spinors. The spinors in ferromagnetic regions are as follows:

$$
\chi_{\sigma}^{(1,3)}=\frac{1}{\sqrt{2}}\left(\begin{array}{c}
1 \\
\sigma i
\end{array}\right)
$$

where $\sigma$ denotes a spin parallel ( $\sigma=\uparrow$ or 1 ) and a spin antiparallel $(\sigma=\downarrow$ or -1$)$ to the magnetization direction
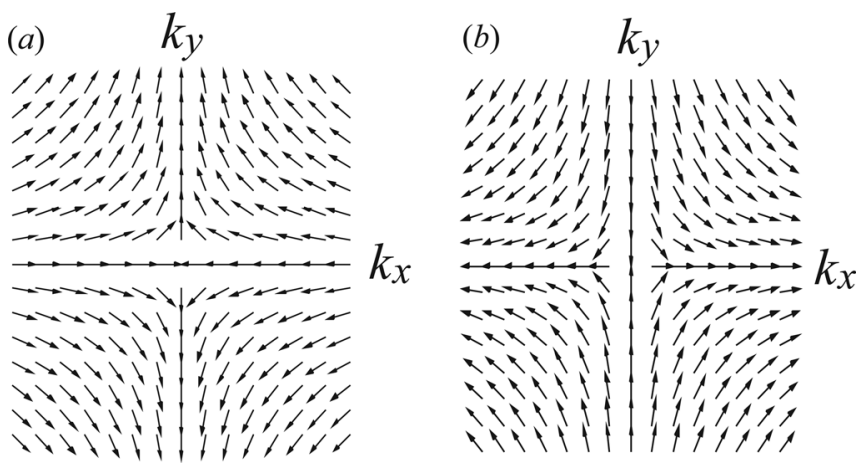

FIG. 2. (a) The spin orientation corresponding to $x_{\sigma+}^{(2)}$ in the $k_{x}-k_{y}$ plane. (b) The spin orientation corresponding to $x_{\sigma-}^{(2)}$ in the $k_{x}-k_{y}$ plane. $\boldsymbol{n}=(0,1,0)$ in the ferromagnets. Therefore, each function $\phi_{\sigma}^{(i)}(z)$ in the regions $1-3$ is as follows:

$$
\begin{aligned}
& \phi_{\sigma}^{(1)}(z)=\frac{1}{\sqrt{k_{z \sigma}}} \exp \left(i k_{z \sigma} z\right) \chi_{\sigma}^{(1)}+r_{\sigma} \exp \left(-i k_{z \sigma} z\right) \chi_{\sigma}^{(1)}, \\
& \phi_{\sigma}^{(2)}(z)=\sum_{j= \pm}\left(A_{\sigma j} \exp \left(i q_{\sigma j} z\right)+B_{\sigma j} \exp \left(-i q_{\sigma j} z\right)\right) \chi_{\sigma j}^{(2)}, \\
& \phi_{\sigma}^{(3)}(z)=t_{\sigma} \exp \left(i k_{z \sigma} z\right) \chi_{\sigma}^{(3)},
\end{aligned}
$$

where $r_{\sigma}, t_{\sigma}$ are spin-dependent reflection and transmission coefficients, respectively. $A_{\sigma \mathrm{j}}, B_{\sigma \mathrm{j}}$ are spin-dependent and subband-dependent transport coefficients. $k_{z \sigma}$ is the wavenumber of electrons with spin $\sigma$ in the ferromagnets $(\mathrm{Fe})$ and $q_{\sigma \pm}$ is the wavenumber in the SC barrier for incoming electrons with spin $\sigma$, given by

$$
q_{\sigma \pm}=\sqrt{\frac{\frac{2 m_{2}^{*}}{\hbar^{2}}\left(E_{\mathrm{F}}-V\right)-k_{\| \sigma}^{2}}{1 \pm \frac{2 m_{2}^{*} \gamma k_{\| \sigma}}{\hbar^{2}}}} .
$$

Taking into account the boundary conditions, we can determine the spin-dependent transmission coefficients. Solving a system of linear equations for $r_{\sigma}, t_{\sigma}, A_{\sigma \mathrm{j}}, B_{\sigma \mathrm{j}}$, we can calculate the transmission probability $T(\theta)$ for the relative angle of $\theta$ between the magnetization directions of the two ferromagnets in the following: $T(\theta)=k_{z \uparrow}\left|t_{\uparrow}(\theta)\right|^{2}+k_{z \downarrow}\left|t_{\downarrow}(\theta)\right|^{2}$. Therefore we can obtain the transmission probabilities $T_{\mathrm{P}}=T(0)$ and $T_{\mathrm{AP}}=T(\pi)$ for parallel and anti-parallel configuration of the ferromagnets. The calculation of the magnetoresistance (MR) ratio has been performed for three different barriers, GaAs $\quad\left(m_{2}^{*}=0.067 m_{e}, \quad \gamma=27.6 \mathrm{eV} \quad \AA^{3}\right)^{13,14}, \quad \mathrm{GaSb}$ $\left(m_{2}^{*}=0.041 m_{e}, \gamma=187 \mathrm{eV}^{3}\right)^{14}$, and GaAs without SOI $(\gamma=0)$ as a function of barrier thickness as shown in Fig. 3 using the following equation: $\mathrm{MR}=\left(G_{\mathrm{P}}-G_{\mathrm{AP}}\right) / G_{\mathrm{AP}}$, where $G_{\mathrm{P}}=\left(e^{2} / h\right) T_{\mathrm{P}}$ and $G_{\mathrm{AP}}=\left(e^{2} / h\right) T_{\mathrm{AP}}$ with $e$ being the elementary charge. The barrier height $V$ is assumed to be $0.75 \mathrm{eV}$ for three different barriers. ${ }^{10}$ From Fig. 3, it is found that the MR ratios show positive values for both GaAs and $\mathrm{GaSb}$ barriers at very thin thickness. Then the MR ratios change signs rapidly from positive to negative with increasing the barrier thickness for both $\mathrm{GaAs}$ and $\mathrm{GaSb}$ barriers. The MR ratio for GaAs barrier remains a negative value with increasing the barrier thickness up to $3 \mathrm{~nm}$. In contrast, the MR ratio for $\mathrm{GaSb}$ barrier changes the sign gradually from negative to positive at a thickness of about $0.9 \mathrm{~nm}$ again. Afterward, its value increases monotonically and the MR ratio reaches to a constant value at a thickness of about $2.5 \mathrm{~nm}$. The MR ratio for GaAs barrier without SOI is shown as a reference for comparison to the case of barriers with SOI. These results of the MR ratios are attributed to the energy splitting in the SC barrier with SOI. Under SOI, the energies of electrons with majority $(\uparrow)$ spin (minority $(\downarrow)$ spin) split into two energies $E_{\uparrow+}, E_{\uparrow-}\left(E_{\downarrow+}, E_{\downarrow \downarrow}\right)$ as shown in Fig. 3(b). Here, for simplicity, we explain the above results with setting $\boldsymbol{k}_{\|}$to be $\left(0, k_{y}\right)$, which does not lose generality. Qualitatively speaking, electrons with higher energies can transmit through the barrier with higher transmission probabilities. In 

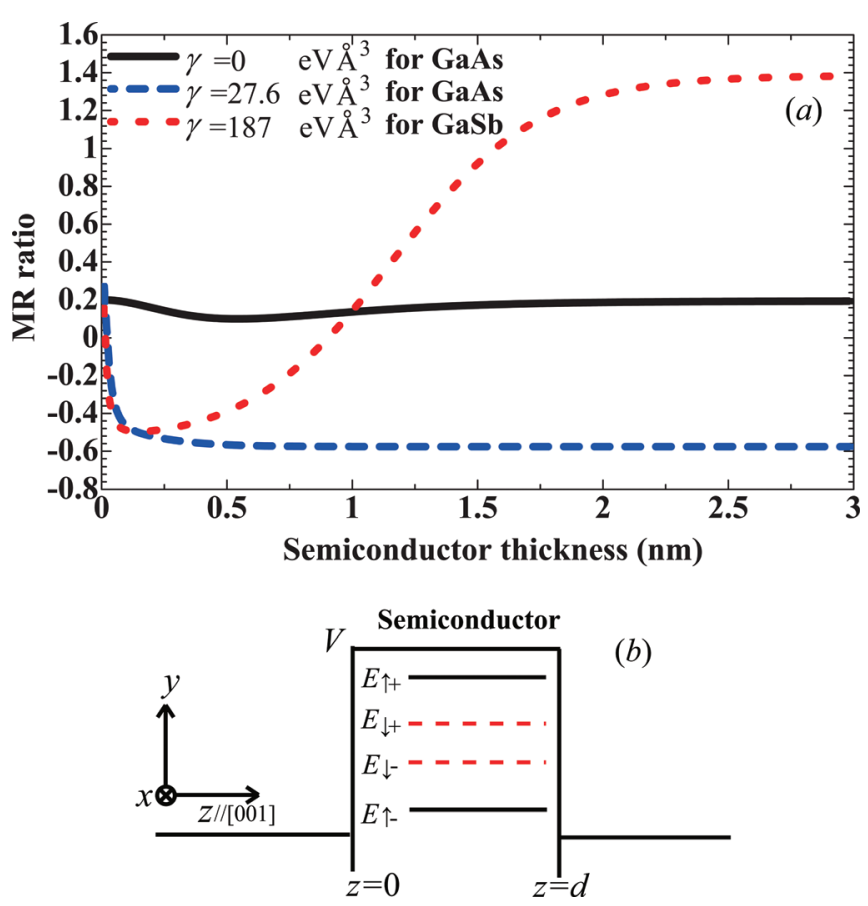

FIG. 3. (Color) (a) The MR ratio for three different barriers, GaAs, GaSb, and GaAs, without SOI as a function of barrier thickness. (b) A schematic illustration of energy levels of electrons with spin $\sigma(\uparrow, \downarrow)$ entering the SC barriers with cubic Dresselhaus SOI.

another point of the view, the direction of spin eigenstates with $E_{\sigma+}$ is parallel to that of majority spin, which is aligned to the positive $y$ axis, and the direction of spin eigenstates with $E_{\sigma-}$ is parallel to that of minority spin, which is aligned to the negative $y$ axis. Therefore the majority (minority) spin in the $E_{\uparrow-}\left(E_{\downarrow+}\right)$ level precesses and flips while transmitting the barrier. The length for the spin-flip is estimated by $\left(\hbar^{2} \pi\right) /$ $\left(2 m_{2}^{*} \gamma k_{z \sigma}^{2}\right)$. In both GaAs and GaSb barriers, electrons are little influenced by SOI at very thin thickness of $0.01 \sim$ $0.02 \mathrm{~nm}$ because the barrier is too thin compared to the Fermi wavelength of $0.6 \mathrm{~nm}$. Therefore, majority electrons with spin $\uparrow$ and minority electrons with spin $\downarrow$ transmit as if the SC barrier was only an insulator without SOI. This results in the positive MR ratio. The SOI starts to affect electrons with increasing the thickness. As a result, minority electrons with spin $\downarrow$ overcome majority electrons with spin $\uparrow$ with increasing the thickness because there are two channels with the second and the third highest energies $\left(E_{\downarrow \pm \pm}\right)$ for spin $\downarrow$. Even though majority electrons in the highest energy $E_{\uparrow+}$ have the highest transmission probability, their probability is slightly smaller than total probability of minority electrons with two channels. This causes the values of the MR ratios to be negative. It is important to note that the spinstates of majority electrons in the highest $E_{\uparrow+}$ are eigenstates, and the spinstates of minority electrons in the second highest $E_{\downarrow+}$ are not eigenstates. Therefore if the spins of minority electrons in the $E_{\downarrow+}$ do not flip, this situation continues. This is the case for GaAs barrier. This is because the length for the spin-flip $((\downarrow) \rightarrow(\uparrow))$ is about $10.253 \mathrm{~nm}$ for GaAs barrier, estimated using the preceding equation. However, this is not the case for GaSb barrier. The length for the spin-flip $((\downarrow) \rightarrow(\uparrow))$ is about $2.42 \mathrm{~nm}$ for GaSb barrier. Then, the value of the MR

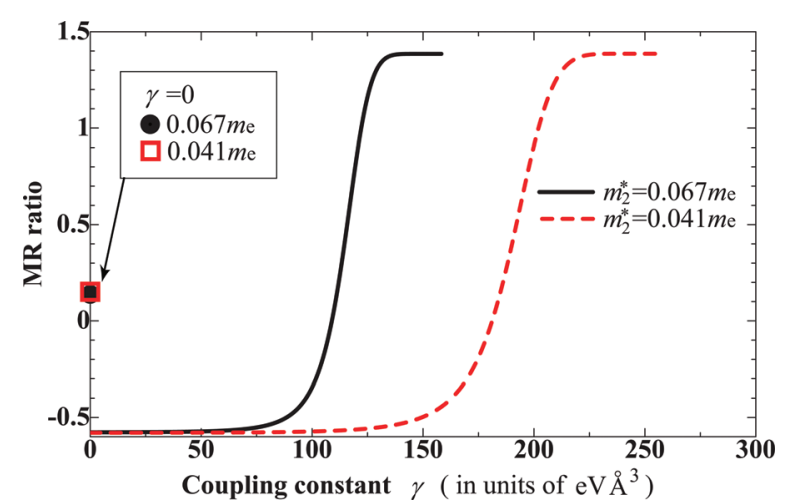

FIG. 4. (Color) The MR ratio for two effective masses of $0.067 m_{e}$ and $0.041 m_{e}$ as a function of spin-orbit coupling constant $\gamma$.

ratio for GaSb starts to change the sign from negative to positive at a thickness of about $1.0 \mathrm{~nm}$, which is about half the value of $2.42 \mathrm{~nm}$. Now, electrons with spin $\uparrow$ occupy the highest and the second highest energies. This causes the value of the MR ratio to be positive with increasing the thickness. Finally, we show the MR ratio for two effective masses of $0.067 m_{e}$ and $0.041 m_{e}$ as a function of spin-orbit coupling constant $\gamma$ in Fig. 4. This calculation has been performed for a barrier thickness of $1.0 \mathrm{~nm}$. Though there is no method to control the value of $\gamma$ at present, it is important to consider $\gamma$ dependence theoretically. In Fig. 4, there is a discontinuity at $\gamma=0$. Therefore the MR ratios at $\gamma=0$ are plotted by the two points (solid circle and open square). When $\gamma$ is small, the situation is the same as that of GaAs. Then the MR ratio is negative. The situation becomes the same as that of GaSb with increasing $\gamma$. This results in the positive value of the MR ratio. Afterward, both the MR ratios for two different effective masses have not been calculated over the specific values $\gamma_{\mathrm{sp}}$. These $\gamma_{\mathrm{sp}}$ 's correspond to values $\hbar^{2} /\left(2 m_{2}^{*} k_{\|}\right)$, which make the denominator $q$ equal to zero. They are estimated to be $158.3 \mathrm{eV} \AA^{3}$ and $258.28 \mathrm{eV} \AA^{3}$ for $0.067 m_{e}$ and $0.041 m_{e}$, respectively. Therefore we have not estimated the MR ratios over the specific values. These results shown in Figs. 3 and 4 suggest that we can control the sign of the MR ratio with increasing the barrier thickness using a material with an adequate $\gamma$. Moreover, these results can provide guidance for material development, such as how much $\gamma$ is required for the desired MR ratio.

${ }^{1}$ G. Autès, J. Mathon, and A. Umerski, Phys. Rev. B 82, 115212 (2010).

${ }^{2}$ S. Honda, H. Itoh, and J. Inoue, J. Phys. D 43, 135002 (2010).

${ }^{3}$ S. Datta and B. Das, Appl. Phys. Lett. 56, 665 (1990).

${ }^{4}$ S. Sugahara and M. Tanaka, Appl. Phys. Lett. 84, 2307 (2004).

${ }^{5}$ S. Kreuzer, J. Moser, W. Wegscheider, D. Weiss, M. Bichler, and D. Schuh, Appl. Phys. Lett. 80, 4582 (2002).

${ }^{6}$ J. Moser, M. Zenger, C. Gerl, D. Schuh, R. Meier, P. Chen, G. Bayreuther, W. Wegscheider, D. Weiss, C.-H. Lai, R.-T. Huang, M. Kosuth, and H. Ebert, Appl. Phys. Lett. 89, 162106 (2006).

${ }^{7}$ K. Kondo and A. Ishibashi, Jpn. J. Appl. Phys. 45, 9137 (2006).

${ }^{8}$ K. Kondo, H. Kaiju, and A. Ishibashi, J. Appl. Phys. 105, 07D522 (2009).

${ }^{9}$ K. Kondo, J. Appl. Phys. 107, 09C709 (2010).

${ }^{10}$ J. Moser, A. Matos-Abiague, D. Schuh, W. Wegscheider, J. Fabian, and

D. Weiss, Phys. Rev. Lett. 99, 056601 (2007).

${ }^{11}$ D. Grundler, Phys. Rev. Lett. 86, 1058 (2001).

${ }^{12}$ M. B. Stearns, J. Magn. Magn. Mater. 5, 167 (1977).

${ }^{13}$ R. Winkler, Springer Tracts Mod. Phys. 191, 74 (2004).

${ }^{14}$ R. de Sousa and S. Das Sarma, Phys. Rev. B 68, 155330 (2003). 